\title{
Computer Simulation of Arcuate and Radial Incisions Involving the Corneoscleral Limbus
}

\author{
K. D. HANNA ${ }^{1,2}$ F. E. JOUVE,${ }^{2}$ G. O. WARING III,${ }^{3}$ P. G. CIARLET ${ }^{4}$ \\ Paris, France and Atlanta, USA
}

\begin{abstract}
Summary
We have created a computer model of the eye that improves upon previous models with two assumptions: (1) the cornea is considered anisotropic, and (2) the strain tensor is non-linear for large displacements. Values used include those for Young's modulus, Poisson's ratio, and three coefficients of anisotropia. A finite element computer program was used to stimulate the behaviour of the eye. Four simulations were done:

(1) a full thickness arcuate limbal cataract incision,

(2) a partial thickness arcuate corneal relaxing incision,

(3) a partial thickness radial incision extending across the limbus,

(4) a partial thickness radial incision confined to the cornea.

A full thickness corneal arcuate incision for cataract induced more change in corneal shape than a limbal incision. However, the gaping is more important in the limbal incision. Partial thickness arcuate corneal relaxing incisions were found to be more effective with a clear zone $3-5 \mathbf{~ m m}$ in diameter.

Radial incision simulation demonstrated little enhancement of the effect of the incisions when across the limbus.
\end{abstract}

The limbus of the eye is the junctional zone between the carefully arrayed lamellar collagen fibrils of the clear cornea and the randomly arrayed collagen fibrils of the opaque sclera. The mechanical function of the limbus originates at the junction of the steeper curve of the cornea with the flatter curve of the sclera. It is a biological junction zone, because the conjunctival stem cells and the aqueous humour trabecular meshwork drainage apparatus are located in this region.

The most common incision made during surgery on the human eye is a limbal incision for both cataract and glaucoma surgery. The limbus is sometimes involved in ocular trauma, particularly in contusion injuries that produce ruptures at the limbus. Therefore, a study of the biomechanics of the corneoscleral limbus is relevant to clinical practice.

Our previous research on computer simulation of the cornea and the globe using a finite element method has revealed the limbus as a region where the stress distribution is different than that on the cornea or the sclera. Specifically, stress increases and changes in the location of the stress-bearing layers, presumably because of the geometry of the limbal sulcus. ${ }^{1-4}$

In this paper, we present a non-linear computer-aided model of the corneoscleral shell using finite element analysis and simulating four types of incisions: 
(1) a perforating arcuate limbal cataract incision,

(2) a non-perforating arcuate corneal relaxing incision,

(3) a non-perforating radial incision that extends across the limbus,

(4) a non-perforating radial incision confined to the cornea.

In addition, we modify our previous finite element models with two new assumptions: (1) the cornea is anisotropic, and (2) nonlinear elasticity is used for large displacements.

\section{Materials and Methods}

We have previously defined the assumptions and methods for this computer model and repeat here only those aspects relevant to this model of the eye and limbus.,

\section{Definition of the Normal Eye}

We used averaged normal values to define the cornea, sclera, and globe. The diameter of the cornea was $11.7 \mathrm{~mm}$. The radii of curvature of the cornea were $7.8 \mathrm{~mm}$ for the anterior surface and $6.6 \mathrm{~mm}$ for the posterior surface. To define and idealise aspheric corneal profile, we used Lotmar's equation for the cornea: ${ }^{5}$

$$
x=\frac{h^{2}}{2 r_{0}}\left[1+a\left(\frac{h}{r_{0}}\right)^{2}+b\left(\frac{h}{r_{0}}\right)^{4}\right]
$$

where $X$ was the profile of the cornea, $r_{o}$ was the radius of curvature of the central cornea, $h$ was the distance to the geometrical axis, and a and $b$ were coefficients, $a=5 / 28$ and $b=1 / 12$. The thickness of the cornea was $0.5 \mathrm{~mm}$ centrally, $\quad 0.74 \mathrm{~mm}$ peripherally, and $0.825 \mathrm{~mm}$ across the limbus. ${ }^{6}$

We defined the sclera as two ellipses with a common axis in order to represent variation in scleral thickness. The external ellipse had a vertical axis $23.48 \mathrm{~mm}$ long and a horizontal axis $22.24 \mathrm{~mm}$ long, while the internal ellipse had a vertical axis $22.74 \mathrm{~mm}$ and a horizontal axis $20.22 \mathrm{~mm}$ long. The thickness of the sclera at the equator was 0.5 and close to the optic nerve was $1.0 \mathrm{~mm}$.

Based on these definitions of the cornea and sclera, we calculated that the volume of the cornea-scleral shell was $917.5 \mathrm{~mm}^{3}$; the inter- nal volume of the eye was $5570.3 \mathrm{~mm}^{3}$; and the axial length of the eye was $23.4 \mathrm{~mm}$ long. This nearly spherical shape seemed acceptable for an emmetropic eye. An intraocular pressure of $15 \mathrm{~mm} \mathrm{Hg}=2000 \mathrm{~Pa}$ was assumed.

\section{Mathematical Relationships, Definitions of Coefficients and Assumptions}

We made three assumptions about the cornea and sclera in this model: (1) Non-linear elasticity is used in order to compute large displacements accurately. (2) The cornea and sclera are essentially incompressible. (3) The cornea is anisotropic. These assumptions are supported in part by previous publications of other authors. ${ }^{7,8}$

We have discussed non-linear elasticity and incompressibility in a previous publication. ${ }^{1}$ We discuss here in more detail the anisotropic behaviour of the cornea and sclera.

An anisotropic tissue is one in which the structure of the tissue is not homogeneous, so that the tissue exhibits a different amplitude of mechanical response when forces are applied from different directions. Anisotropic behaviour would be assumed for the cornea based upon its lamellar structure. The collagen fibrils form relatively discrete lamellae from anterior to posterior (including Bowman's layer and Descemet's membrane) and extend from one location on the limbus to another. There are no published studies quantifying the anisotropic behaviour of the cornea. However, Battaglioli and $\mathrm{Kamm}^{7}$ demonstrated in sclera that the elastic modulus for perpendicular compressive stress was 100 times less than the elastic modulus for circumferential tangential stress. The general principles of anisotropic behaviour have been described by Green and Zerna as 'transversal isotropia', in which five elastic coefficients are used to describe the behaviour of a tissue. ${ }^{9}$

We have used these principles in constructing a computer model of the cornea that includes three coefficients of anisotropia (a1, a2, a3) to the tissue. The coefficients of anisotropia are ratios of response of the cornea to forces applied in two directions, the normal direction perpendicular to the surface and the tangential direction parallel to the surface. These ratios express the unequal response of the tissue in these two different 
directions. A ratio of one means that the response is the same in the two directions; the smaller the ratio, the greater the difference in response in the two directions.

The first coefficient of anisotropia, a1, designated the ratio of the elongation of a cube of cornea when the same traction force is applied in the perpendicular and the tangential directions. We assigned a value of 0.01 to this based upon the findings of Battaglioli ${ }^{7}$ in the sclera, an approximation. This means that the corneal tissue elongates 100 times less in the tangential direction parallel to the lamellae than in the normal direction perpendicular to the lamellae. The second coefficient of anisotropia, a2, was a ratio of displacement of a cube of cornea, between the displacement in a perpendicular direction when submitted to a traction force in the tangential direction and the displacement in the tangential direction when submitted to the same force in the perpendicular direction. The value for this coefficient was 0.10 , based upon theoretical computation. The third coefficient, a3, designated the sheer induced by torsional forces and is the ratio of sheer response to torsional force applied in both the perpendicular and the tangential directions. We assigned a value of one to this coefficient, because the value was indeterminable based upon known information about the cornea.

We have selected a value of 4860 Kilo-Pascals for Young's modulus (E), based upon the work of Woo and colleagues ${ }^{10,11}$ and a value of 0.49 for Poisson's ratio (v) based upon the work of Battaglioli. ${ }^{7}$

Knowing that the structure of the limbus is different from that of the cornea and on the assumption that collagen fibrils at the limbus have a circular or arcuate direction, ${ }^{4}$ we assumed the limbus to be 150 times stronger in the circular direction around the limbus than in the direction perpendicular to the surface of the limbus. This assumption produced the following values for the limbus: Young's modulus $=100$ Kilo-Pascals, in the perpendicular direction, and $15000 \mathrm{KPa}$ in the tangential direction Poisson's ratio $=0.49$, and the coefficients of an anisotropia a $1=150$, $\mathrm{a} 2=1$, and $\mathrm{a} 3=1$. The assumed values for the sclera are a Young's modulus of 2000 KiloPascals and Poisson's ratio of 0.49 .
In selecting these values, we were aware that the true value for each variable is unknown and that these represent approximations.

Using these values, we calculated a stressstrain relationship where

$\Sigma=\left[\begin{array}{l}\sigma_{1} \sigma_{6} \sigma_{5} \\ \sigma_{6} \sigma_{2} \sigma_{4} \\ \sigma_{5} \sigma_{4} \sigma_{3}\end{array}\right]$ and $\varepsilon=\left[\begin{array}{l}\varepsilon_{1} \varepsilon_{6} \varepsilon_{5} \\ \varepsilon_{6} \varepsilon_{2} \varepsilon_{4} \\ \varepsilon_{5} \varepsilon_{4} \varepsilon_{3}\end{array}\right]$ are respectively the stress and strain tensors expressed in a basis where the first vector is parallel to the direction of anisotropia. Then we have the stress-strain relationship:

$$
\begin{aligned}
& \sigma_{1}=a_{1} \frac{E(1-v)}{(1+v)(1-2 v)} \varepsilon_{1}+\frac{E v}{(1+v)(1-2 v)}\left(a_{2} \varepsilon_{2}+a_{2} \varepsilon_{3}\right) \\
& \sigma_{2}=\frac{E(1-v)}{(1+v)(1-2 v)} \varepsilon_{2}+\frac{E v}{(1+v)(1-2 v)}\left(a_{2} \varepsilon_{1}+\varepsilon_{3}\right) \\
& \sigma_{3}=\frac{E(1-v)}{(1+v)(1-2 v)} \varepsilon_{3}+\frac{E v}{(1+v)(1-2 v)}\left(a_{2} \varepsilon_{1}+\varepsilon_{2}\right) \\
& \sigma_{4}=\frac{E}{2(1+v)} \varepsilon_{4} \\
& \sigma_{5}=\frac{E}{2(1+v)} a_{3} \varepsilon_{5} \\
& E L 7 \sigma_{6}=\frac{E}{2(1+v)} a_{3} \varepsilon_{6}
\end{aligned}
$$

Where E is the Young's modulus $v$ is the Poisson's coefficient $a_{1}, a_{2}, a_{3}$ are the three 'coefficients of anisotropia".

\section{Finite Element Method Computer Program}

As discussed in standard texts, ${ }^{12,13}$ the finite element method studies a structure by dividing it into a finite number of elements or 'bricks' connected to each other by nodes. The nodes form a grid or mesh. It is assumed for each brick element that only the value of the displacement at each of the nodes is needed to define the displacement for the whole element. In this way it is easy to describe mathematically the response of a single brick element to a force and, based on the total number of elements, to define the response of the entire structure, such as the cornea. Increasing the number of brick 
elements and the number of nodes per element increases the accuracy of the model.

We use the finite element method in a three-dimensional model with curved elements. All computations were done using 618 elements in half of the eye, assuming symmetry of the globe. We assigned more brick elements to the central cornea and to the limbus, since these were the areas of displacement in which we were the most interested. We utilised two elements across the thickness of the model.

We used ten steps of approximation, with 22,507 equations for each step. The amount of computer memory needed to calculate the solution for these equations was more than 100 megabytes and approximately 45 minutes per step were required using an IBM 4381 computer.

We think this model is an improvement over the previous models we have published. ${ }^{1,2}$

\section{Tissue Response Simulated}

Our model simulated the instantaneous change induced by an incision immediately after the forces changed by the incision had reached equilibrium. The model did not take into account delayed processes such as the viscoelastic mechanical response of the tissue or changes induced by wound healing. The computations allowed measurement of change over the entire surface of the globe. We report here the findings concerning the cornea and the limbus.

\section{Simulated Arcuate Incision for Cataract Surgery}

We studied two types of arcuate incisions used for cataract surgery, one in the limbus and one in the cornea. Both incisions were 120 degrees long and were made perpendicular to the surface of the globe as a single full thickness perforating incision. The limbal incision was located at a diameter of $12 \mathrm{~mm}$ which would approximate the 'blue zone' of the surgical limbus and the corneal incision was made at a diameter of $10.4 \mathrm{~mm}$, which would approximate the tip of the limbal vascular arcade.

We assumed that the intraocular pressure fell from $15 \mathrm{mmHg}$ to $3 \mathrm{mmHg}$ after the full thickness incision and the computations were based on the pressure of $3 \mathrm{mmHg}$. We report the displacement of the central cornea and the amount of gaping of the wound induced by this incision.

We also simulated a nonperforating arcuate limbal incision as would be performed in cataract surgery, but to a depth of nearly 100 per cent, so that only the three basal nodes of the brick elements persist. This allowed modelling of the changes in the cornea while the intraocular pressure was still $15 \mathrm{mmHg}$, as opposed to the circumstance when the incision was perforating and the pressure fell to $3 \mathrm{mmHg}$.

\section{Arcuate Non-perforating Corneal Relaxing Incisions for Astigmatism}

We simulated two arcuate, 60 degrees long, perpendicular incisions of the cornea placed across both sides of the $0-180$ degrees meridian. All of the incisions were made on a model eye that was assumed to have 5.4 diopters of preoperative astigmatism, with a total refractive power for the eye of 51.2 diopters at 90 degrees and 56.6 diopters at 0-180 degrees (against the rule astigmatism). Each incision severed all of the nodes in the model except for the three nodes at the base of each posterior brick element, so that the incision was just short of 100 per cent of the thickness of the cornea. Incisions were located at both sides of six zone diameters $3.2,4.8,6.9,8.0$ and $9.6 \mathrm{~mm}$. We also simulated placement of four incisions, two at zone 7.2 and two at zone 8.8. The specific diameters were selected to coincide with the space between elements, which did not always fall on a whole number diameter. We report the displacement of the central cornea induced by the incisions and the change in dioptric power of the cornea using Gullstrand's optical model of the eye. ${ }^{14}$

\section{Linear Radial Nonperforating Incisions for the Correction of Myopia}

We simulated the effect of two types of radial keratotomy, using eight total incisions, those that crossed the limbus (from zone $3.2 \mathrm{~mm}$ to $14.0 \mathrm{~mm}$ ) and those confined to the cornea (from zone 3.2 to $11.5 \mathrm{~mm}$ ). These incisions penetrated all of the cornea except for the last remaining nodes on each brick element. We report the displacement and change in 
dioptric power of the central cornea induced by each of these incisions.

\section{Stresses Studied}

As we have described in detail previously, ${ }^{1,2}$ we describe the influence of the meridional stress (the stress tangent radially at each point from the centre of the cornea to the optic nerve), the hoop stress (the stress tangent circumferentially at each point of the cornea parallel to the limbus), and the compressive stress (the stress acting perpendicularly to each point of the corneoscleral shell). The three sheer stresses were studied, but are not represented in the results because their value was minimal.

\section{Results}

\section{Stress Distribution Before Surgery}

The findings in this anisotropic model were different and more refined than those in the previously presented isotropic model, where stress distribution decreased gradually from
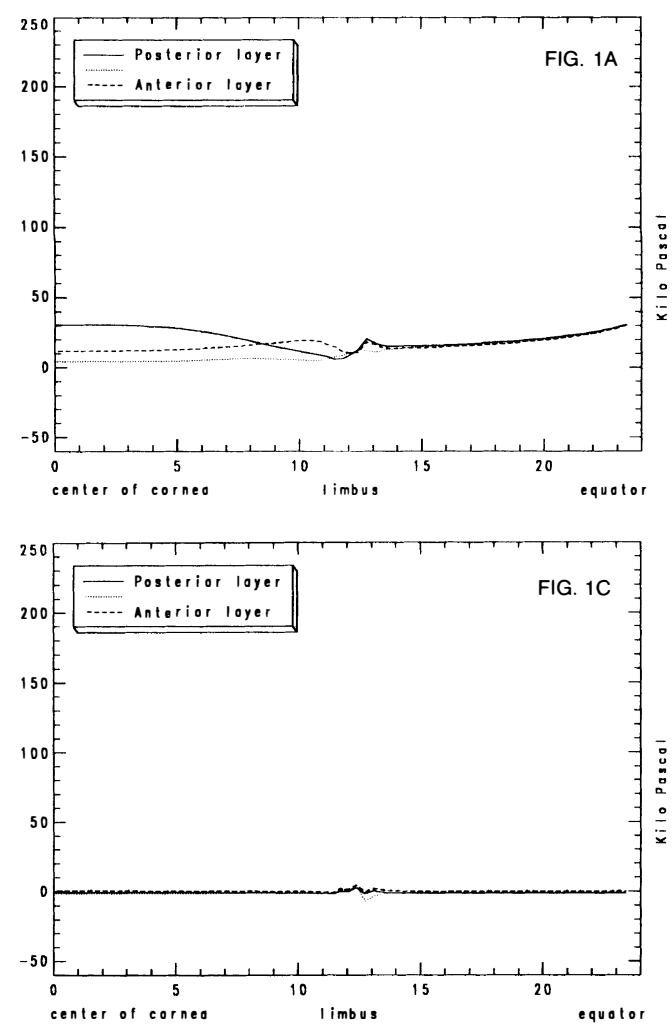

the posterior to the anterior surface of the cornea. In the anisotropic model, there was less stress borne by the middle layers of the cornea (the mid-stroma) than by the anterior layer for the three types of stress. For meridional and hoop stresses, the changes in the peripheral cornea and the limbus began at a diameter of approximately $6 \mathrm{~mm}$ and extended out to $13-14 \mathrm{~mm}$. For meridional stress, the anterior layers of the cornea bore more stress in the limbal area (Fig. 1a). For hoop stress, the posterior layers bore the most stress across the peripheral cornea and limbus (Fig. 1b). For the perpendicular normal stress, the values are very small and the layers are approximately equal in their support (Fig. 1c). The patterns of stress across the cornea and limbus showed a striking similarity: the stresses on all layers of the cornea became the same at approximately a diameter of $12 \mathrm{~mm}$. Presumedly, this point of uniformity, which might represent the exact point of the biomechanical limbus, resulted from the changes in curvature of the cornea and sclera meeting

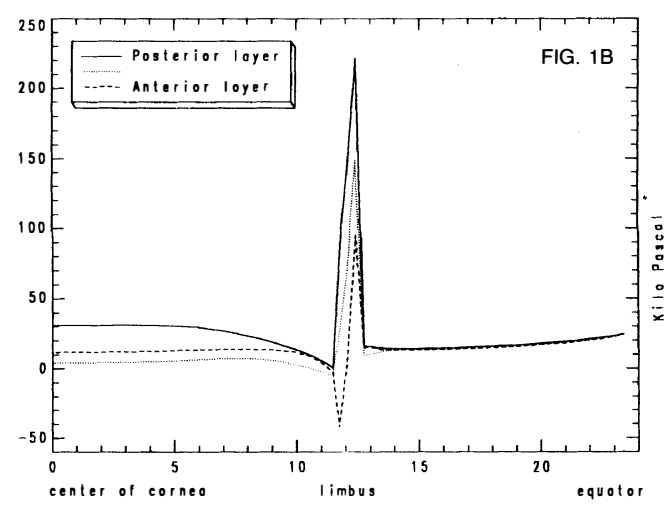

Fig. 1. Non-linear anisotropic model of stresses on the cornea, limbus and anterior sclera before surgery. Graphic representation demonstrates the stress in kilo-pascals on the vertical axis, the location of the stress in terms of millimeters from the centre of the cornea along the horizontal axis, and the stress at three layers (posterior, middle, and anterior) of the tissues. (a) Meridional stress is borne more by the posterior layers centrally and is borne equally by all layers at the limbus. (b) The hoop stress is borne more by the posterior layer centrally and showed increase across the limbus. (c) The compressive stress shows minor changes in distribution across the cornea limbus and sclera. 
and forming the limbal sulcus, a point where we have assumed a change in mechanical properties by assigning different values to the cornea on one side and the limbus to the other as described above. Outside of this zone at 12 to $14 \mathrm{~mm}$, the hoop stress showed a very large inflection, in which this portion of the limbus supports four times the stress of the cornea or the sclera, presumedly emphasising its structural role and also reflecting the assumptions made in constructing this model. All three stresses are nearly equal in the sclera.

Sheer stresses were absent in the cornea and the sclera and small amplitude sheer stresses were observed in the limbal area.

Against this background of preoperative normal findings, we investigated the effects of various types of incisions.

\section{Arcuate Incision for Cataract Surgery}

Non-perforating Limbal Incision: The arcuate incision made at a diameter of $12 \mathrm{~mm}$, non-perforating, produced minor variations in meridional stress in the peripheral cornea
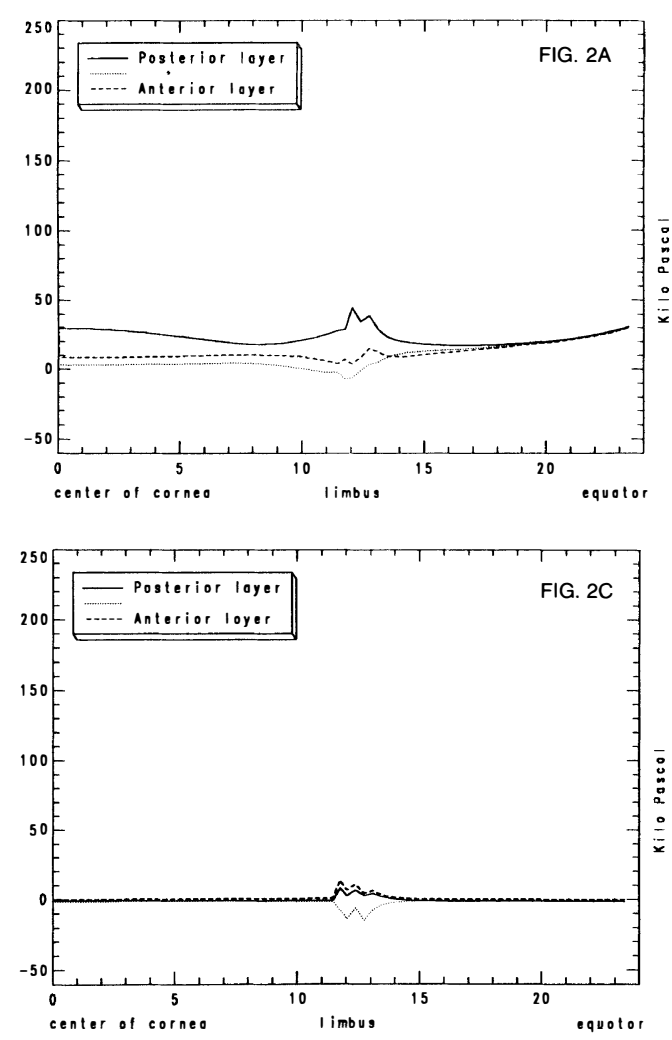

and limbus (Fig. 2a). In contrast, there were major fluctuations in the hoop stress at the point of the incision (Fig. 2b). The hoop stress rose six times in the residual posterior layer in the area of the incision. There was major alteration in the compression stress (Fig. 2c).

An incision made at $13.5 \mathrm{~mm}$ in the diameter in the sclera induced virtually no change in the cornea.

Arcuate Incision in the Peripheral Cornea, Non-perforating: The meridional stress began to increase all layers in the paracentral and peripheral cornea, rising from about $5 \mathrm{~mm}$ out to the $10.4 \mathrm{~mm}$ location of the incision, where it fell back to the preoperative value at $12 \mathrm{~mm}$ in the limbal zone, a negative value for the median layer 'compressive function' (Fig. 3a). The hoop stress also began to change at about $5 \mathrm{~mm}$ with the posterior and anterior layers bearing more stress and this increasing out to the point of the incision at $10.4 \mathrm{~mm}$, where the stress fell off markedly in the area of the wound and then rose to very high values in the posterior layer at the limbus at $12-13 \mathrm{~mm}$

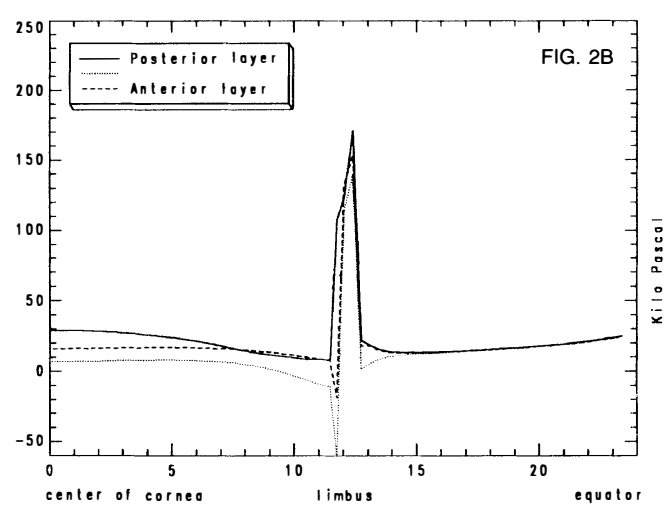

Fig. 2. Effect of a 120 degrees non-perforating incision placed at $12 \mathrm{~mm}$ diameter. (a) the meridional stress is increased in the area of the incision at the limbus primarily with some increase at the peripheral area of the cornea. (b) The hoop stress shows a drop in the peripheral cornea and a major change in the middle layers with a negative increase in the stress. (c) The perpendicular compressive stress also becomes higher in the middle layers at the limbus in the area of the incision. 

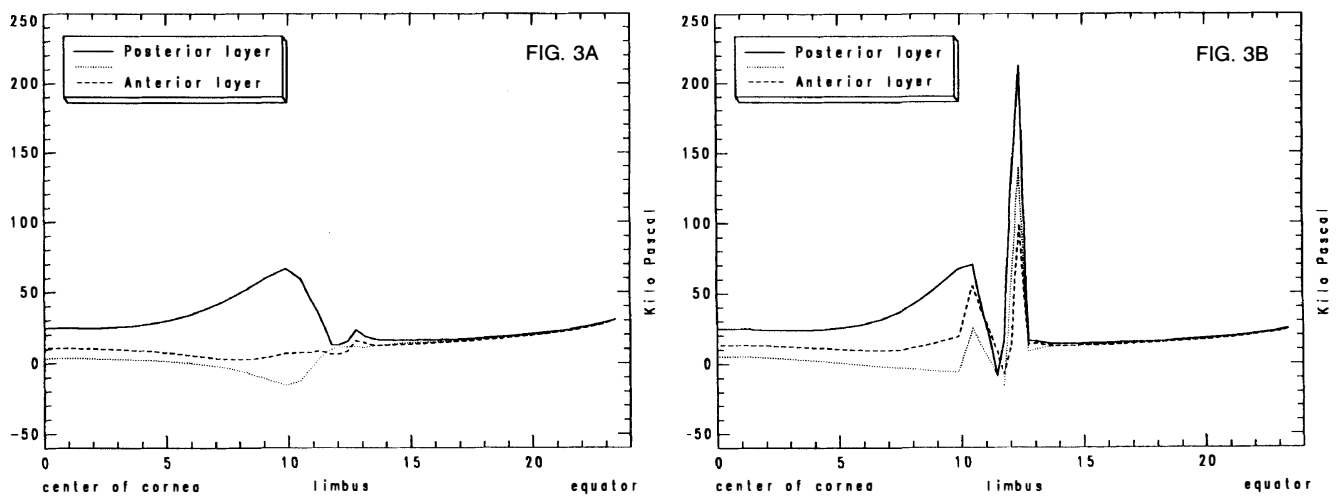

Fig. 3. Effect of a 120 degrees non-perforating incision placed at a diameter of $10.4 \mathrm{~mm}$. (a) Meridional stress shows a marked increase in the peripheral cornea and in the area of the incision, with middle layer showing a negative inflection. (b) The hoop stress also shows an increase in the peripheral cornea with a major inflection in the area of the incision.

(Fig. 3b). Thus, the corneal incision had much more effect on peripheral corneal shape than the limbal incision.

Perforating Limbal and Corneal Arcuate Incisions: It was difficult to produce curves of the distribution of stress across the layers of the cornea and limbus after a perforating wound, because the intraocular pressure dropped to near atmospheric levels. Our simulation assumed an intraocular pressure of $3 \mathrm{mmHg}$. Thus, the major force producing these stresses was very small. Therefore, to simulate the biomechanical results of these incisions, we studied the displacement of the anterior and posterior layers of the central cornea as well as the amount of gaping in the middle part of the arcuate incision. The incision at the limbus at a $12 \mathrm{~mm}$ diameter produced a posterior displacement of the surface of the cornea of $.074 \mathrm{~mm}$ (74 microns) whereas the incision in the cornea at $10.4 \mathrm{~mm}$ diameter produced a posterior displacement of the central cornea of $0.1 \mathrm{~mm}$ (100 microns). However, the gaping of the wounds was different: limbal incision $=$ anterior surface, $0.23 \mathrm{~mm}$ and posterior surface: $0.21 \mathrm{~mm}$; corneal incision = anterior surface, $0.13 \mathrm{~mm}$ and posterior surface, $0.11 \mathrm{~mm}$.

We interpret these findings in the following way. There is greater gaping at the limbus than in the cornea because of the geometry of the limbus and because we have assumed that the limbus is stiffer than the cornea. There is less displacement, however, by the limbal incision because the stress concentrated at the limbus is not transmitted to the central cornea as readily as the stress in the area of the corneal incision itself, so the corneal incision will produce a greater central flattening than will the limbal incision. (Figs. 4 and 5).

In the computer model, we also simulated a rise of intraocular pressure to $15 \mathrm{mmHg}$ after the perforating incisions and found that the limbal incision gaped more than the corneal incision, as might be expected from a rise in intraocular pressure during cataract surgery.

\section{Radial Non-perforating Incisions}

We compared the effect of eight non-perforating radial incisons confined to the cornea with those extending across the limbus. The meridional stress response was similar in both circumstances, (Figs. 6a and 7a) the stress on the posterior layer doubling in the central cornea and falling to zero at approximately $12 \mathrm{~mm}$ at the limbus, except the incisions extending across the limbus created variations in the stress out into the sclera as far out as the equator.

The hoop stress showed the expected large increase in stress at the limbus in the posterior layers, but the incisions confined to the cornea created an increase in stress in the anterior layers of the limbus whereas those crossing the limbus created a major increase in the stresses, including a marked negative value for the middle layers, suggesting wide gaping of the wound as it crossed the limbus (Figs. 6b 

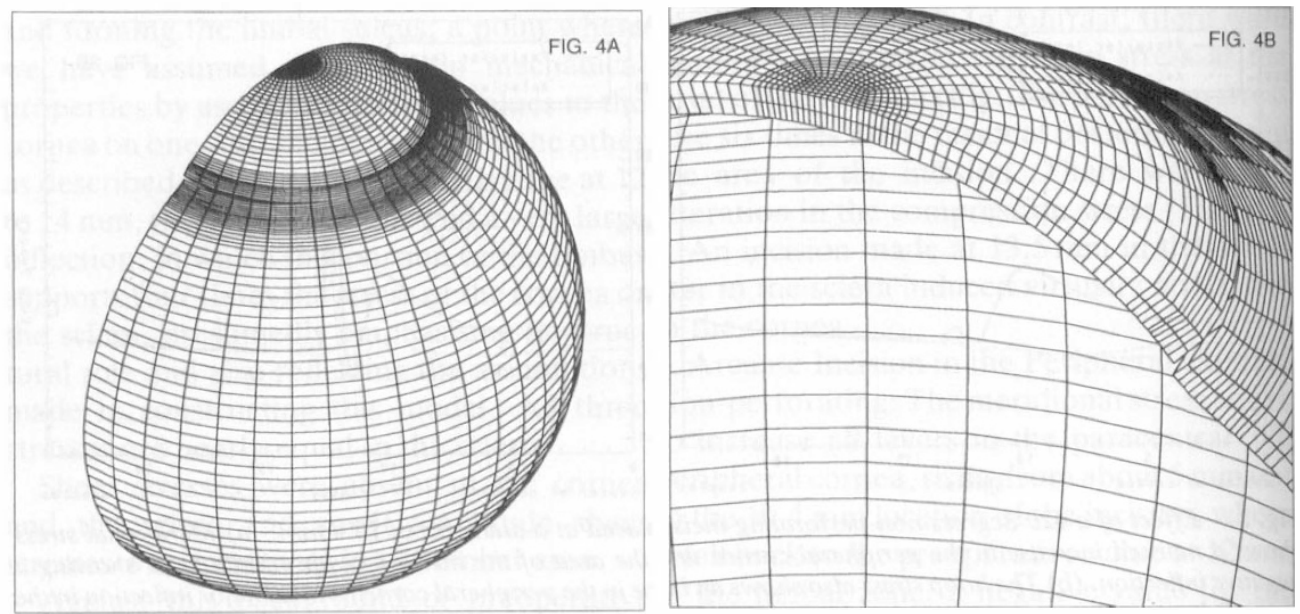

Fig. 4. Effect of 120 degrees, perforating incision made at $12 \mathrm{~mm}$ diameter through the limbus. Three-dimensional model of the eye shows gaping of the limbal wound with some flattening of the central cornea and slight anterior displacement of the limbus just inside of the wound. (a) View from outer portion of whole eye. (b) Cross sectional view of cornea and limbus.

and $7 b$ ). As expected, the compressive stress and the sheer stresses showed little change.

When we computed the difference in refractive effect based on the displacement of the central cornea, we found a change of 3.25 diopters for the corneal radial incisions and 3.60 diopters for the incisions that crossed the limbus, a difference of 0.35 diopters. This modelling demonstrates negligible increase in effect with incisions across the limbus.

\section{Non-perforating Corneal Arcuate Incisions for Astigmatism}

We studied the effect of 60 degrees long arcuate non-perforating corneal incisions for astigmatism in two ways; first by studying the change in stress patterns on the three layers of the cornea and second by studying the displacement and change of refractive power of the central cornea. There was an increase in meridional stress in the posterior layer and a small negative value in the stress of the middle layers with almost no change in the anterior layers in the area where the incision was made, while there is only a small change in the central cornea. (Fig. 8).

Figure 9 demonstrates the change in central corneal refractive power with incisions placed from 3.2 to $9.6 \mathrm{~mm}$ diameter zones. Along the 0-180 degrees meridian, there is a flattening of the central cornea when the incisions are placed from approximately 3 to $5 \mathrm{~mm}$, but this effect becomes less from approximately 6 to $8 \mathrm{~mm}$ and is negligible by the time the zone reaches $9 \mathrm{~mm}$ in diameter. The overall amount of flattening in this meridian is approximately 1.5 to 2 diopters for the 3-5 mm zone incisions. The 90 degrees meridan shows steepening of approximately 1 diopter, and interestingly, this 1 diopter of

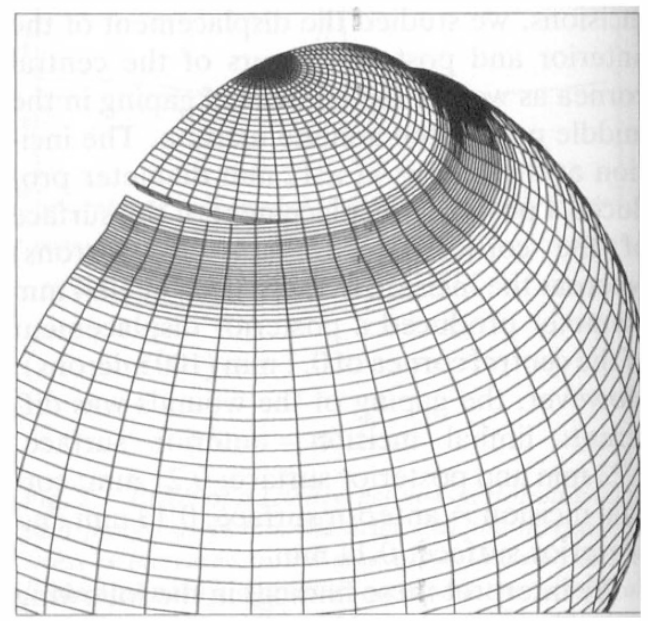

Fig. 5. Effect of 120 degrees, perforating incision made at $10.4 \mathrm{~mm}$ diameter in the cornea. Model of the eye viewed exteriorly shows gaping of the wound, slight anterior displacement of the cornea on the central side of the wound and flattening of the central cornea. 

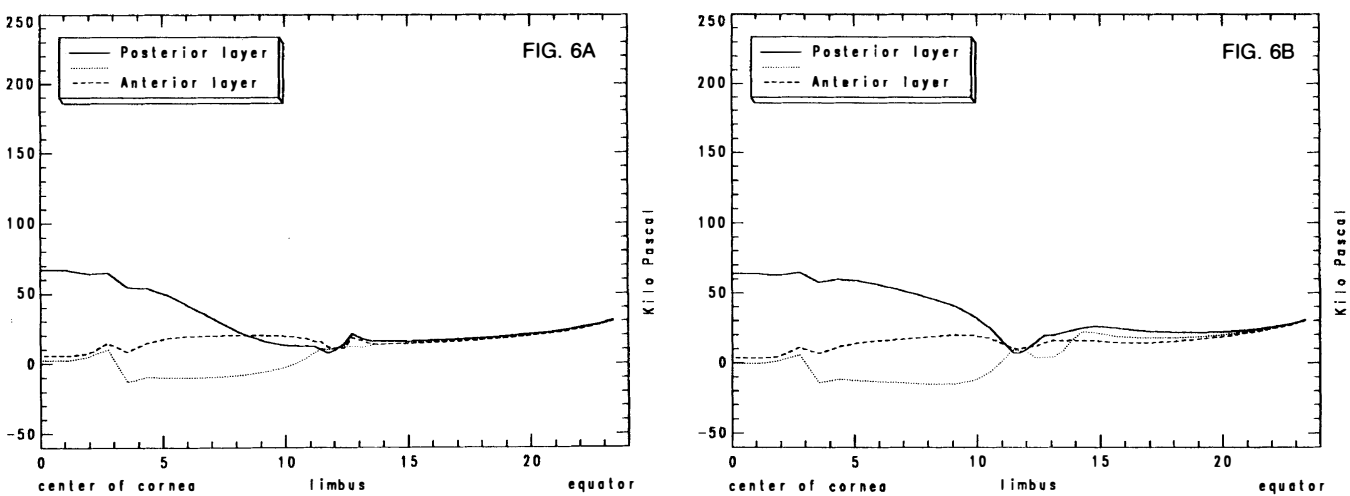

Fig. 6. Effect of eight radial incisions confined to the cornea (total diameter $11.7 \mathrm{~mm}$ ). (a) Meridional stress shows increased stress on the posterior layers in the central cornea, diminishing to the limbus and a negative compressive stress on the middle layers. (b) Hoop stress shows an increase in stress on the posterior layers centrally and a decrease in stress on the middle layers paracentrally, the stress being equilibrated at the limbus at $11 \mathrm{~mm}$, with the limbal spike being present as in the normal cornea.

steepening is consistent from the 3 to $9 \mathrm{~mm}$ zone incisions. These values are quantified in the Table.

The stimulation of four incisions, two at diameter 7.2 and two at diameter $8.8 \mathrm{~mm}$ showed an expanded area of increased stress in the posterior layer that ranged from a diameter of approximately 5 to $10 \mathrm{~mm}$.

The results in the Table are similar to those achieved clinically where an effect of approximately 1 to 3 diopters can be achieved with this type of incision.

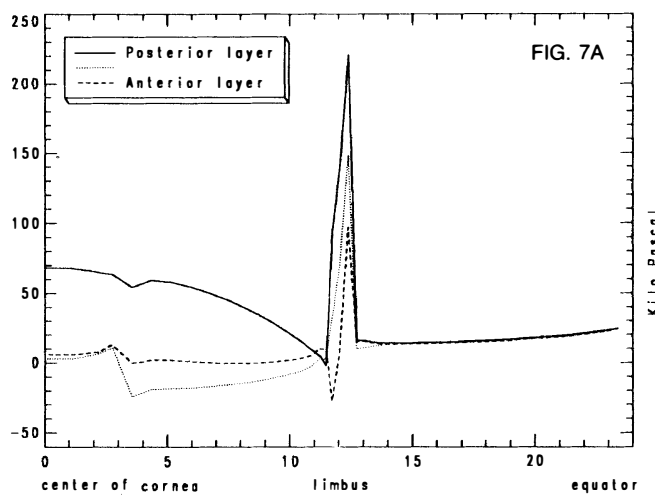

In all cases, the stresses became the same and close to the zero baseline value at $12 \mathrm{~mm}$ diameter at the limbus, emphasising the role of the limbus as a barrier.

\section{Discussion}

Our attempts at computer modelling of refractive corneal surgery have evolved through three phases with increasing sophistication. The first phase was a linear isotropic model of lamellar keratectomy. ${ }^{2}$ The second was a non-linear, isotropic model of radial

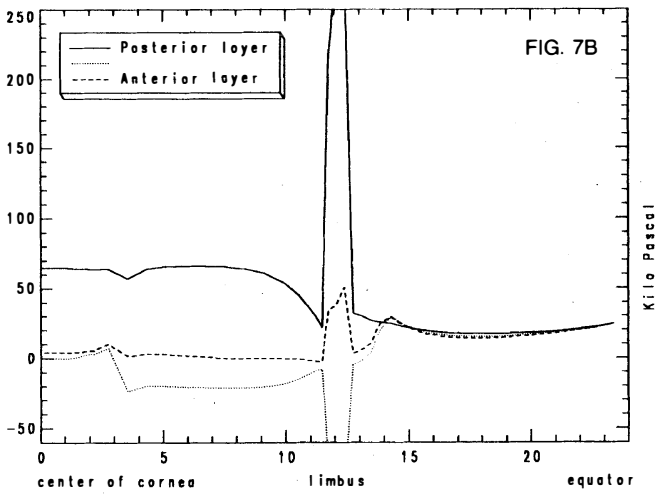

Fig. 7. Effect of eight radial incisions, non-perforating, extending across the limbus for a total diameter of $14 \mathrm{~mm}$. (a) Meridional stress shows an increase in the posterior layer centrally and in the peripheral cornea with a decrease in the paracentral and peripheral stress in the middle layers. There is change in the stress across the limbus and into the sclera out to the equator which is not present in incisions confined to the cornea. (b) Hoop stress shows an increase in the central and paracentral cornea across the posterior layers and a marked increase across the limbus in the posterior layers. The decrease in the paracentral and peripheral cornea in the middle layers is apparent, but becomes great across the limbus as well, indicating major change in stress across the limbus induced by the incisions and the spreading of the wounds. 

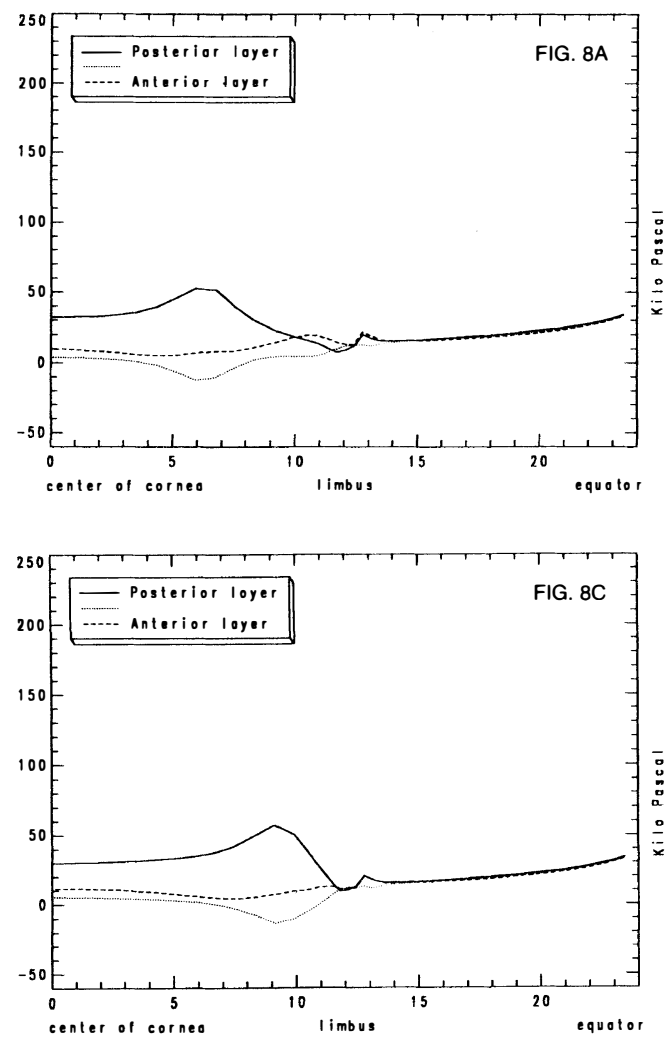

incisions. ${ }^{1}$ The third and present model is a non-linear (for large displacements), anisotropic model of both arcuate incisions parallel to the limbus and radial incisions.

\section{General Observations Concerning the Limbus From the Model}

Modelling the baseline and postoperative changes demonstrated that the limbus plays a special role, partially because of its unique geometry as the junction between the two different radii of curvature of the cornea and the sclera creating the limbal sulcus and partially because of the assumptions in the model that the limbus is 100 times stiffer in the circumferential direction than in the radial direction. These two factors combine to make the limbus a zone of change in distribution of the value of stresses, and this protects the cornea from rupture. This is the biomechanical role often conceived of the limbus by clinicians and surgeons.

These special properties of the limbus, as contrasted to the central cornea or the sclera,

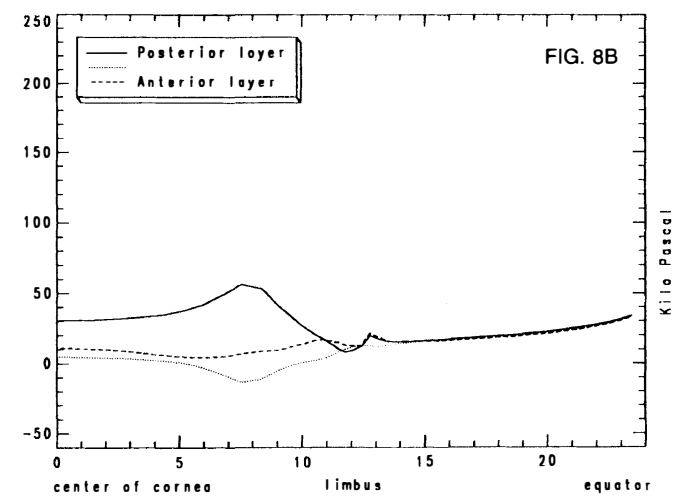

Fig. 8. Effect of 60 degrees arcuate non-perforating corneal incisions for astigmatism on meridional stress. (a) Incision at $6.4 \mathrm{~mm}$ diameter shows increased stress in the posterior and middle layers in the area of the incision. (b) Incision at $8 \mathrm{~mm}$ shows similar increase in spread of stress. (c) Incision at $9.6 \mathrm{~mm}$ shows increased stress in the area of the incision. In $a, b$ and $c$, stress is equilibrated at the limbus.

make the limbus stiffer and the adjacent area more fragile so that increased stresses such as a large rise in intraocular pressure from blunt trauma are not distributed as easily in the limbus as they are elsewhere in the globe, predisposing the area close to the limbus to rupture, a circumstance observed clinically.

The stress values for the anterior, middle, and posterior layers often come together as a single value at the limbus in the simulations that we performed, emphasising the role of the limbus as a stiffer tissue (where the stress distribution is the same) and across all the limbus tissues (where it is different across the cornea).

The major modifications in hoop stress shown for all of our simulations are a function of the biomechanical assumptions made in the model, and need to be verified.

The six types of stresses that we studied (meridonal, hoop, compressive, and three types of sheer) responded differently under different circumstances.

The meridional stress in the central cornea 

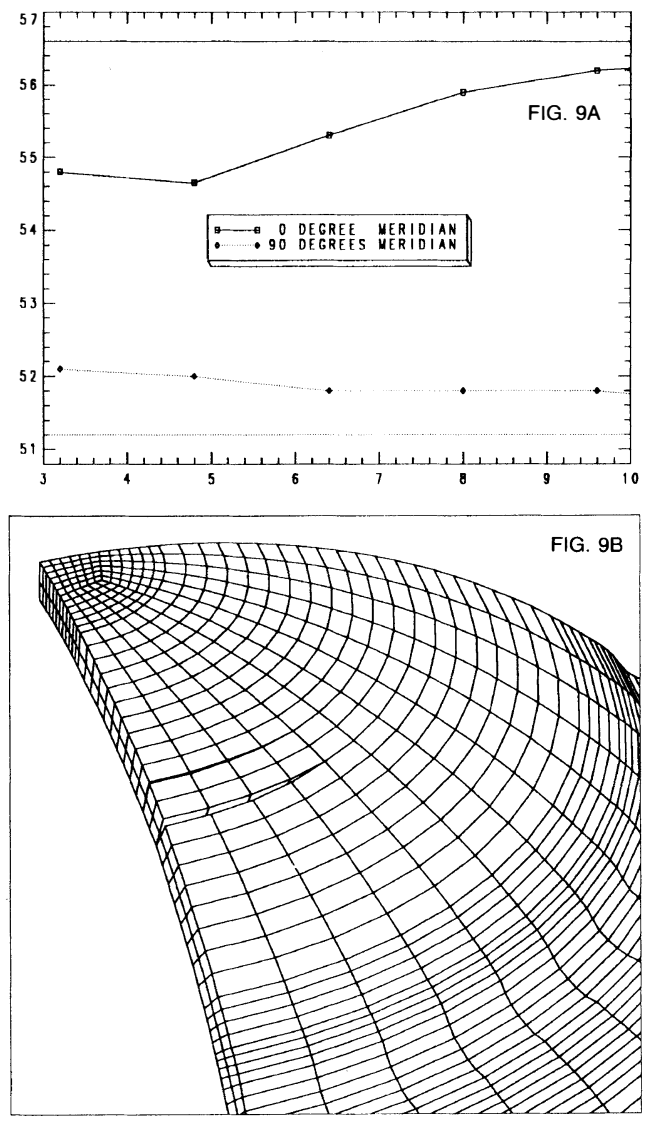

Fig. 9. (a) Effect of two arcuate 60 degrees long nonperforating relaxing incisions in the cornea. Incisions are made perpendicular to the 0-180 degrees meridian (solid line and boxes) and approximately parallel to the 90 degrees meridian (dotted and triangles). The baseline value in this simulated eye was 6 diopters of astig. matism for the entire refractive power of the eye or 56.6 diopters in the 0 degrees meridian and 51.2 diopters in the 90 degrees meridian. The vertical axis shows the refractive power of the whole eye. The horizontal axis shows the diameter separating the location of the two arcuate incisions. Incisions made from 3 to $5 \mathrm{~mm}$ have marked flattening effect in the 0 degrees meridian and some steepening effect in the 90 degrees meridian. Incisions from 5 to $7 \mathrm{~mm}$ have less effect but still a clinically meaningful one. Incisions from 7 to $9.5 \mathrm{~mm}$ have increasingly less effect on the change in corneal curvature. (b) Diagram showing effect of 60 degrees nonperforating corneal relaxing incision.

shows the greatest change after eight radial incisions, simply reflecting the impact of the number of incisions on the biomechanical structure of the cornea. However, the eight radial incisions create minimal change at the limbus. On the other hand, arcuate incisions create less change in the meridional stress centrally, but produce large changes in the stress in the area in which the incision is made, whether it be in the cornea or at the limbus.

The changes in hoop stress at the central cornea are similar, being somewhat large in the eight radial incisions and small in the arcuate incisions, but the changes in hoop stress at the limbus are very large in both circumstances, particularly in the exact area where the incisions are made.

The changes in compressive stress and sheer stresses are negligible in the face of these incisions, because there is not much alteration in the force applied across the cornea itself or parallel to the corneal surface under the circumstances simulated here.

\section{Assumptions}

Any computer model is based on assumptions that require verification under experimental and clinical circumstances. The major asssumptions in our model are two-fold. The first are the assumptions of the material properties of the cornea, including Young's modulus, Poisson's ratio, and the coefficients for anisotropia. We have used the published values in the literature and reasonable estimates in our model, but until experimental studies can more reliably quantify these properties in human corneas, the field of computer modelling will be dependent upon approximations only.

The second set of assumptions are the role of non-linear and anisotropic conditions in the cornea. The published stress-strain curves for the cornea and sclera demonstrate that for small displacements, changes are approximately linear, but that as the displacements become larger, non-linear relationships become more evident. ${ }^{15}$

A third premise on which our results are based is simply that the values represent the acute change after the incision and do not take into account either the viscoelastic, timedependent changes in the tissue, or the changes due to wound healing over time.

In spite of these assumptions, the findings of the model approximate those achieved in clinical practice. This means that computer modelling, as it becomes increasingly refined, 
Table. Effect of arcuate non-perforating paracentral and peripheral 60 degrees corneal incisions made perpendicular to the 0-180 degrees Meridian

\begin{tabular}{cccccc}
\hline & \multicolumn{2}{c}{$0-180$ degrees Meridian } & & \multicolumn{2}{c}{90 degrees Meridian } \\
\cline { 2 - 3 } \cline { 5 - 6 } $\begin{array}{c}\text { Diameter of zone } \\
\text { for incision }\end{array}$ & $\begin{array}{c}\text { Total refractive } \\
\text { power of the eye } \\
(D)\end{array}$ & $\begin{array}{c}\text { Change from } \\
\text { baseline } \\
(D)\end{array}$ & & $\begin{array}{c}\text { Total refractive } \\
\text { power of the eye } \\
(D)\end{array}$ & $\begin{array}{c}\text { Change from } \\
\text { baseline } \\
(D)\end{array}$ \\
\hline Baseline & 56.6 & & & & 0 \\
3.2 & 54.8 & -1.8 & & 51.2 & +0.9 \\
4.8 & 54.65 & -1.95 & & 52.1 & +0.8 \\
6.9 & 55.3 & -1.38 & & 51.8 & +0.6 \\
8.0 & 55.9 & -0.7 & & 51.8 & +0.6 \\
9.6 & 56.2 & -0.4 & & 51.8 & +0.6 \\
$7.2+8.8$ & 55.7 & -0.9 & & 51.9 & +0.7 \\
\hline
\end{tabular}

may allow the surgeon to perform the operation on the computer screen and predict the outcome, giving him the flexibility to modify parameters in the model and select those that might be most appropriate for an individual patient. Of course, further refinements will be necessary before this clinical advantage can be realised.

\section{Incisions Simulating Cataract Surgery}

When we compared arcuate incisions in or parallel to the limbus that might be used in cataract surgery, those confined to the cornea produced greater displacement and change in stress in the peripheral cornea than those confined to the limbus. This correlates with clinical experience, where surgeons find that corneal incisions are more likely to induce astigmatism after cataract surgery than limbal or scleral incisions.

\section{Radial Non-perforating Incisions}

The simulation of radial incisions using a nonlinear anisotropic model was different than that we published previously using a non-linear isotropic model. ${ }^{1}$ In the anisotropic model, the anterior and posterior layers behave similarly to the situation in the isotropic model, particularly the posterior layer which shows a larger change in stress centrally after the incisions and that change in stress diminishes as one approaches the limbus. However, the middle layers show little change in a negative stress in the anisotropic model.

Our simulation of radial incisions confined to the cornea versus those extending across the limbus are consistent with clinical experi- ence and the early models of Schachar ${ }^{16}$ where little enhancement of the effect of the incisions across the limbus is achieved. We have demonstrated previously that a change in the length of the incision of $0.5 \mathrm{~mm}$ produced a change of approximately 15 per cent of the correction, and that the incisions extending from a clear zone diameter greater than $4 \mathrm{~mm}$ had a decreasing effect. Simulations with this anisotropic model supported that observation.

\section{Arcuate Non-perforating Corneal Incisions for Astigmatism}

Our major observation concerning corneal arcuate incisions for astigmatism concern the effect of the location of the incisions. Those made at a zone of 3 to $5 \mathrm{~mm}$ had the most flattening effect in the meridian perpendicular to the incision and the most steepening (coupling) effect in the meridian 90 degrees away. In the zones from approximately 5 to $7 \mathrm{~mm}$, this effect diminished in both meridians and from $7 \mathrm{~mm}$ to $9.5 \mathrm{~mm}$, there was minimal effect from the incisions. We did not simulate the different lengths of these arcuate incisions.

\section{Conclusion}

We have presented a non-linear, anisotropic model of the eye with an emphasis on the biomechanical role of the limbus in surgical incisions performed during cataract surgery, radial keratotomy and astigmatism surgery. The limbus plays a vital biomechanical role as demonstrated by this model, because of its unique geometry and presumed greater stiff- 
ness. Further refinements of the model in terms of the mechanical properties of these tissues, the role of non-linear anisotropic factors, and ultimately the influence of time dependent viscoelastic and wound healing effects will be necessary to allow clinical application.

\section{References}

${ }^{1}$ Hanna KD and Jouve FE: Preliminary computer simulation of the effects of radial keratotomy. Arch Ophthalmol. (In Press).

${ }^{2}$ Hanna KD, Jouve FE, Bercovie JH, Waring GO: Computer simulation of lamellar keratectomy and myopic keratomileusis. J Refrac Surg. (In Press).

${ }^{3}$ Maurice DM: The cornea and sclera. In Davson $\mathrm{H}$ ed. The Eye. Vol.1, 3rd ed., London: Academic Press 1985; 1-158.

${ }^{4}$ Maurice DM: Mechanics of the cornea. In Cavanagh HD ed. The Cornea: Transactions of the World Congress on the Cornea III, New York: Raven Press 1988: 187-193.

${ }^{5}$ Lotmar W: Theoretical eye model with aspherics. $J$ Optical Soc Am 1971; 61: 1522-9.

${ }^{6}$ Duke-Elder S and Wybar KC: Anatomy of the visual system. In Duke-Elder S ed. System of Ophthalmology. Vol. II. St Louis: CV Mosby 1961; 92-120.
${ }^{7}$ Battaglioli JL and Kamm RD: Measurements of the compressive properties of the scleral tissue. Invest Ophthalmol Vis Sci 1984; 25: 59-65.

${ }^{8}$ Chuong $\mathrm{CHJ}$ and Fund YC: Compressibility and constitutive equation of arterial wall in radial compression experiments. J Biomechanics 1984; 17: $35-40$.

${ }^{9}$ Green AE and Zerna W: Theoretical elasticity. 2nd ed. London: Oxford Press 1968: 159.

${ }^{10}$ Woo SL, Kobayashi AS, Schlegel WA: Non-linear material properties of intact cornea and sclera. Exp Eye Res 1982, 14: 29-39.

${ }^{11}$ Woo SL: Structural analysis of a cornea-scleral shell. Seattle, Washington: Doctoral Thesis, University of Washington, 1971.

12 Ciarlet PG: The finite element method for elliptic problems. New York: North Holland Publishing 1978: 36-101.

${ }^{13}$ Zienkiewicz OC: The finite element method. 3rd ed. New York: McGraw Hill 1977.

${ }^{14}$ Gullstrand A: Helmholtz's treatise on physiological optics. Vol. I. 3rd ed. Southall JPC (trans). New York: Optical Society of America 1924.

15 Taber LA: Large deformation mechanics of the enucleated eyeball. ASME J Biomech Eng 1984; 16: 229-35.

${ }^{16}$ Schachar RA, Black TD, Huang T: A physicist view of radial keratotomy with practiced surgical implications. In Schachar RA, Levy NA, Schachar L eds. Keratorefraction. Denison, TX: LAL Publishing 1980: 195-220. 